\title{
Bolívar Echeverría: el discurso crítico y la política de la forma natural
}

\section{Bolívar Echeverría: Critical Discourse and the Politics of the Nature Form}

\author{
Andrea Torres Gaxiola \\ Universidad Nacional Autónoma de México, México \\ atorresgaxiola@gmail.com
}

Resumen: En este artículo se presentan las implicaciones políticas y críticas que supone el estudio del valor de uso en la obra de Bolívar Echeverría. El marxismo clásico se ha centrado a lo largo del tiempo en el análisis de distintos elementos propios de la teoría de Marx, como los modos de producción, la relación entre estructura-superestructura, la teoría del valor, la teoría de la crisis, entre otros. Sin embargo, en muy pocos casos se ha destacado la importancia del valor de uso y sólo se han esbozado algunas ideas derivadas de este concepto. Una notable excepción es $E l$ discurso crítico de Marx, donde Echeverría propone volver al estudio del valor de uso con el fin de replantear la discusión de lo político dentro del marxismo. Con base en ello, en este artículo se intenta mostrar la hipótesis teórica de Bolívar Echeverría en la que propone reinterpretar la obra de Marx a partir del eje forma natural-valor de uso como mecanismo para replantear el discurso crítico hacia una mayor comprensión de aquello que corresponde a la esfera de lo político. Para Echeverría el ámbito en el que se encuentra la esencia de lo político y la autarquía de lo humano es la de la reproducción social de la vida, es decir la de la construcción concreta de la forma natural. 
Palabras clave: valor de uso, forma natural, política, teoría crítica, marxismo latinoamericano.

Abstract: In this article, we present Bolivar Echeverría's use value theory and its implications. Clasical Marxism has long centered the study of Marx's theory on modes of production, the relationship between structure-superstructure, the value theory, the crisis theory, among others subjects. However, it has rarely focused on Marx's use value concept and only few ideas have been devised upon it. A noticeable exception is $E l$ discurso critic de Marx (Marx's critical discourse), where Bolivar Echeverría proposes to go back to capital's essential contradiction: use value vs. value, and to formulate a use-value theory. Thus, this paper presents the critical and political implications of Echeverria's theory: based upon the core idea natural form-use value, he establishes a critical discourse that is able to understand capitalism politics and presents us with a better comprehension of the field of politics. For Echeverría, rather than the traditional understanding of politics in terms of the super-structure of economics, the human political life takes place in the reproduction of social life, in the concrete construction of a nature-form.

Keywords: Use value, Nature Form, Politics, Critical theory, Latin-American Marxism.

Recibido: 28 de junio de 2019 Aprobado: 10 de septiembre de 2019 https://dx.doi.org/10.15174/rv.vi25.485 
Las unicas formas reales de las mercancías son sus figuras de uso, sus formas naturales.

Karl Marx, Capítulo IV inÉdito

$\mathrm{B}^{1}$ concepto de valor de uso, una las determinaciones de la mercancía, ha sido una categoría olvidada por el marxismo. Sin embargo, el desarrollo del capitalismo ha mostrado lo importante que resulta destacar dicha categoría en el contexto de este modo de reproducción social. El valor de uso hace referencia a espacios que dentro del discurso de la economía se encuentran completamente velados: el consumo de bienes. Hoy en día, este espacio y su relación con la forma de producción y reproducción en la sociedad contemporánea es fundamental, y en efecto, autores como Kojin Karatani, André Gorz y John Bellamy Foster, ${ }^{1}$ entre otros, han destacado que el regreso al análisis de la forma natural, a los modos de consumo y la relación entre la producción, la circulación y el consumo son el camino hacia un nuevo planteamiento de la discusión de lo político dentro del discurso marxista contemporá-

${ }^{1}$ Estos autores han tratado de retomar la obra de Marx a partir de un esfuerzo de renovación de la interpretación que gira en torno al problema del valor de uso (el cual sin duda engloba el problema de la circulación de bienes, de la naturaleza como la fuente de la riqueza y el consumo como la esfera de satisfacción de las necesidades humanas). Por ejemplo Kojin Karatani propone cambiar el enfoque de lectura hacia los "modos de intercambio" y la circulación del capitalismo frente a las tradicionales lecturas que se han centrado en los modos de producción y la esfera de la producción del capital (véase Karatani, 2004). John Bellamy Foster, por su cuenta, propone realizar una lectura a partir de la relación entre el capitalismo y la naturaleza, resaltando una de las consecuencias más funestas del capitalismo: la fractura del metabolismo entre el hombre y la naturaleza (John Bellamy, 2004) Por su parte, André Gorz, propone centrarse en los modos de consumo que son consecuencia de la producción capitalista y que resultan en una dependencia de la sociedad del capitalismo (Gorz, 2011). 
neo. Estos autores destacan, a grandes rasgos, que lo político no sólo se juega en el proceso de producción industrial del capital, sino que está también en juego en el proceso de circulación, de consumo de la riqueza capitalista. Todos estos intentos recientes pretenden destacar que la dimensión de lo político no sólo está centrada en el ámbito de la política como parecería estar situado en el capitalismo, esto es, en el estado, sino que se ubica tanto en el ámbito del consumo, de la circulación, como en el de la producción mercantil.

Bolívar Echeverría es uno de los primeros autores que observa cómo en El capital se analiza el valor de uso como momento de la dimensión de lo político. Lo hace en su primer libro libro sobre Marx: El discurso crítico de Marx. Se trata de uno de los intentos más originales de renovar el discurso marxista. Echeverría parte del desarrollo de la teoría de la subsunción de Marx para extenderla hacia la esfera de la forma natural: no sólo es el proceso de producción el que está subsumido al mando del capital, ${ }^{2}$ sino que "la forma social-natural" concreta se ve subsumida a la forma de reproducción capitalista, la cual es abstracta y autoimplicante. En este artículo nos proponemos hacer una lectura de su obra para mostrar la relación que Echeverría establece entre la crítica como discurso y la política como su objeto a partir de la reformulación de los conceptos de valor de uso y forma natural que en Marx quedó apenas esbozado. De modo que la cuestión que aquí nos planteamos será la siguiente: ¡en qué sentido la forma natural de Marx es planteada en la obra de Bolívar Echeverría como la categoría crítica central, descartada en gran medida por la tradición, y

${ }^{2}$ Con base en la teoría de la subsunción del trabajo al mando del capital, Echeverría propone extender dicha tesis a la totalidad del proceso social, incluyendo el ámbito mismo de lo político. Dicha propuesta permite reformular la ya superada teoría de lo político entendido en términos de la "superestructura" determinada por la estructura económica o la base material (ver Marx, 2001). 
que, sin embargo, se presenta como la categoría a desarrollar? Para ello, debemos, en un primer momento, establecer de qué modo se define la crítica en el discurso de Echeverría, en un segundo momento, presentar brevemente un recorrido por el concepto de valor de uso dentro del marxismo y, para terminar, desarrollar la forma que adopta dicha teoría en la obra de Bolívar Echeverría.

\section{La función de la crítica}

Para el filósofo ecuatoriano, volver a establecer los términos del discurso crítico de Marx significaba recordar el sentido de su filosofía crítica, tarea que realiza en su libro El discurso crítico de Marx. En efecto, tomando como base la línea teórica de la Escuela de Frankfurt, para Echeverría todo discurso crítico debe siempre ser negativo: debe minar la lógica del discurso positivo, es decir, del discurso imperante. Su obra comienza con la constatación del siguiente hecho: "nunca como en el siglo xx tantas posibilidades sociales y técnicas de felicidad, de armonía entre los hombres, y entre éstos y la naturaleza fueron convertidas de manera tan sistemática en compulsiones a la desgracia y a la destrucción” (Echeverría, 2017: 17). Esta es la clave para la comprensión del siglo xx, en el cual la humanidad ha escogido el camino de la barbarie en detrimento del socialismo, y en consecuencia se ha esmerado en demasía en hacer uso de la razón, en justificar, en crear conocimiento para destacar "el aspecto positivo" de ésta. En cambio, la teoría que se ha identificado como "de izquierda" y "marxista", al parecer se vio agotada a finales del siglo xx. En efecto, la crisis del marxismo se debió principalmente a su incapacidad de poner en tela de juicio los principios del discurso imperante; en particular en lo que respecta al cuestionamiento del carácter técnico y productivista propio de la sociedad capitalista o de plantear un discurso negativo frente al neoliberalismo que surge a finales del siglo xx. 
En este sentido, Echeverría propone volver a plantear los términos de la discusión en la que se despliega la teoría crítica marxista. Para ello, destaca dos aspectos con los cuales Marx define la tarea de una filosofía crítica: el primero, del joven Marx, la de realizar en términos teóricos la "encomienda comunista". Esta tarea que implica la adopción de una postura política radical consiste en "reconocer la posibilidad inminente del fin del ser humano" (Echeverría, 2017: 21). El segundo aspecto, consiste en el reconocimiento de la tesis central del Marx tardío: el hecho de que todas las contradicciones, los conflictos y desgracias de la sociedad actual se reducen a una contradicción fundamental y fundacional: la del valor de uso frente al valor, es decir, la contradicción que se inserta en la estructura misma de la riqueza del capital.

Con base en este reconocimiento el filósofo ecuatoriano se aventura a realizar un replanteamiento de la politicidad básica del ser humano. Se trata de releer la obra de Marx a partir de un eje que lo guía: su carácter crítico está centrado en la capacidad de redefinir el "ámbito de lo humano", en tanto que "dimensión político-genérica", como una dimensión enajenada que el ser humano debe recuperar, que, a su vez, se presenta como la causa del absurdo de la sociedad contemporánea. La hipótesis de Echeverría es la siguiente: todo discurso teórico que se pretende crítico debe necesariamente plantearse la tarea de comprender la auténtica dimensión de la politicidad humana sin reservas y sin concesiones. Lo fundamental de la sociedad capitalista como una forma civilizatoria en la que prevalece la barbarie y la ausencia de sentido radica en el hecho de que lo político como dimensión humana ha sido enajenada al pseudo-sujeto capitalista. Al respecto Echeverría sostiene: "las posibilidades de una nueva forma de vida social es idéntico a hablar de las imposibilidades de la vida social en su forma dada, la forma capitalista". Por lo tanto, se trata de: "desestructurar el discurso establecido, el discurso burgués capitalista, 
del que debe partir ineludiblemente" (2017: 253) De modo que la crítica se define no como un discurso que "bosqueje una teoría socialista" sino como un discurso que deconstruya el discurso de la economía política. En el "ámbito de lo propiamente humano", se trata de desestructurar la forma en que éste, lo político, se presenta bajo el discurso del capitalismo, esto es, desestructurar la idea de que lo político sólo pertenece a ciertas instituciones tales como el Estado y la nación capitalistas.

De modo que nuestro autor parte de una hipótesis de interpretación de la obra de Marx en los siguientes términos: "La aprehensión cabal del mensaje marxiano que, se supone, es lo que pretende cualquiera de sus textos, resulta ser una meta casi imposible de alcanzar". En efecto, la obra de Marx, en tanto que una obra inacabada, siempre se nos presenta como incompleta y por momentos contradictoria. Echeverría continúa:

Si en la obra de Marx hay un texto principal porque en él está la clave de los demás y si éste es inconcluso porque quedó aún en proceso de alcanzar su versión definitiva, la única lectura adecuada que se puede hacer de ella es la que, al asumir la problematicidad, se convierte necesariamente en un co-escribirla. Leer a Marx, resulta así, llevando las cosas al extremo, emprender la tarea paradójica de escribir, junto con él, su propia obra.

Así, la estrategia heurística de Echeverría radica en la idea de que, para aprehender el "mensaje marxiano", es necesario reconstruir su obra. Esto es lo que hace en El discurso crítico de Marx. La limitación de los planteamientos del marxismo ortodoxo se debe a que toman la obra de Marx como un edificio inamovible y, a su vez, incuestionable; como un sistema completo, suficiente y cerrado. En consecuencia, Echeverría retoma un concepto que es clave en El capital, pues es en su problematicidad, es decir, en la con- 
tradicción intrínseca del capitalismo, donde debe encontrarse el núcleo del discurso crítico. Esta contradicción consiste, de acuerdo con el Marx de El capital, en el siguiente hecho: el valor, en tanto que medida abstracta de la riqueza, sólo puede manifestarse por medio de su contrario, el valor de uso, la forma natural concreta del proceso social; de modo que la problematicidad central del discurso de Marx está en el hecho de que toda forma natural, producto de este modo de producción social, estará siempre subsumida por su forma antitética, el valor, por un principio abstracto de estructuración de lo social. La estrategia heurística es entonces la siguiente: para recuperar el ámbito de lo político, que está ausente en el capitalismo, habrá que replantear la obra de Marx a partir de una lectura guiada por la categoría de la forma natural. Para Marx, el valor de uso no sólo significa el aspecto útil de una mercancía, sino que involucra una forma concreta de organización social, que, a su modo de ver, siempre se refiere a una multiplicidad histórica en su modo de concreción. Es a partir de este principio teórico que Echeverría se propone, a lo largo de su obra, realizar una teoría de la "forma natural".

\section{El valor de uso y la tradición marxista.}

En la obra de Marx, la introducción de la categoría del valor de uso como una característica fundamental de la economía, significó la reintroducción de un elemento despreciado por la tradición económica, en la cual ya de por sí existía una gran confusión entre el concepto de valor y el concepto de valor de uso. Por ejemplo, al criticar a Ricardo y a Say en los Grundrisse, Marx sostiene:

¿pero este contenido en cuanto tal, no se desarrolla hasta formar un sistema de necesidades y producción? ¿El valor de uso, en calidad de tal, no se introduce en la propia forma, no la determi- 
na económicamente, por ejemplo, en la relación entre capital y trabajo? ¿En las diversas formas de trabajo? [...] sea como fuere, hay que investigar esto concienzudamente al estudiar el valor, y no como hace Ricardo, dejarlo sencillamente de lado, ni como el insulso Say darse ínfulas con el mero empleo de la palabra 'utilidad'. Ante todo, se debe exponer y se expondrá en el desarrollo de los diversos capítulos, en qué medida el valor de uso en cuanto sustancia presupuesta queda al margen de la economía y de sus determinaciones formales y en qué medida entra ellas (Marx, 1973: 206).

Pero ¿qué implica poner en el centro de la discusión al valor de uso y a la forma natural en la lectura que realiza Echeverría sobre la obra de Marx, en particular en su lectura de El capital? Significa, como bien sostiene Marx en los Grundrisse, cuestionar si el capitalismo, como forma de producción y consumo social, es capaz de satisfacer las necesidades de los individuos. De modo que el problema de lo político en el capitalismo gira en torno a la relación entre las necesidades humanas y la forma en que éstas se producen y reproducen.

Este cuestionamiento tiene cierta historia, que a continuación revisaremos brevemente. Ya en las "Glosas marginales al tratado de economía política de Adolph Wagner”, Marx (1976) se esfuerza por mostrar que la sustancia social no se reduce a la sustancia del valor, la cual es, a grandes rasgos, el tiempo socialmente necesario del trabajo, sino que lo social en tanto que "sustancia" es la mercancía, es decir, la relación que emana de la forma mercantil. Esta idea es esencial en la discusión dentro del marxismo, pues lo social, en consecuencia, no está sólo determinado por la forma en la que se presenta en capital, como valor, sino que se trata de un proceso de subsunción de "lo social" como forma de organización concreta, una forma de organización abstracta que se guía por la lógica 
del valor; esta idea es la que Echeverría pretende destacar en su interpretación de El capital. La tradición marxista dio por sentado esta categoría en tanto que un simple presupuesto del valor. Para Paul Sweezy, por dar un ejemplo, el valor de uso no es relevante pues no develaba ninguna relación social (Ortega Reyna, 2014). ${ }^{3}$ Rodolsky (1968), en cambio, sí dedica tiempo a dicha categoría, sin por ello llegar a un análisis completo de la forma natural; no obstante, muestra que el valor de uso resulta fundamental en la medida en que es determinante para entender el desarrollo mismo de la forma mercantil.

A su vez, la Escuela de Frankfurt reconoce la importancia de esta categoría. Marcuse, en Razón y Revolución sostiene:

la introducción de la categoría de valor de uso era la introducción de un factor olvidado, y esto por la economía política clásica, que únicamente se había ocupado del fenómeno del valor de cambio. Poniendo de nuevo en el centro la categoría del valor de uso, se somete al proceso económico a una aguda interrogación en el sentido de si satisface, y cómo, las necesidades de los individuos (1972: 297). ${ }^{4}$

Esta categoría cuestiona y revela, a su vez, la contradicción entre la lógica del valor que mueve a la economía y la finalidad inmediata de lo social: el consumo y la reproducción social. Continúa Marcuse: "Tras las relaciones de cambio en el capitalismo, esta

${ }^{3}$ En este artículo Jaime Ortega realiza un recorrido teórico detallado del concepto de valor de uso tanto en Bolívar Echeverría como en la tradición marxista. Véase también el artículo de Daniel Inclán, Márgara Millán y Lucia Linsalata (2012) "Apuesta por el valor de uso: aproximación a la arquitectónica del pensamiento de Bolívar Echeverría".

${ }^{4}$ A pesar de destacar el valor crítico de dicha categoría, Marcuse no avanza hacia una teoría del valor de uso y de la forma natural. 
categoría muestra las relaciones humanas efectivas, uncidas a una 'totalidad negativa' y regidas por leyes económicas incontrolables" (298). En sus términos, el valor de uso como concepto le permite mostrar a Marx en qué medida el proceso de reproducción capitalista es uno que es "ciego, azaroso, anárquico y de frustración" (298).

Alfred Schmidt, por su cuenta, es quizás el primer autor en realizar una teoría de la forma natural en el sentido de que se propone realizar un estudio del concepto de naturaleza en la obra de Karl Marx. Se cuestiona por qué razón Marx deja de lado el análisis de la forma natural en favor de la forma de valor:

Marx tiene que desatender la forma natural de las mercancías, su valor de uso, exactamente en la medida que es característica de la economía burguesa. En el capitalismo, la naturaleza que el hombre se ha apropiado aparece como mero 'soporte material' del valor de cambio, lo único determinante; sus cualidades humanizadas, la posibilidad que le es inmanente de satisfacer necesidades de hombres vivientes, es algo externo al proceso de valorización del capital (Schmidt. 1975: 32).

Este autor subraya lo determinante de la forma natural como concepto: en primer lugar, esta categoría refiere a un ámbito dejado de lado por la economía política: el del consumo y el disfrute, es decir, el del mundo de los valores de uso en tanto que satisfactores de necesidades. En segundo lugar, en el capitalismo, el valor de uso aparece como un elemento prescindible, a pesar de que es el soporte de la forma de valor. Es decir, si bien para la lógica del valor el valor de uso se presenta como un "pretexto" necesario para la valorización del capital, éste es imprescindible, y cuando el capital olvida dicho hecho, por lo general entra en crisis, crisis de acumulación, crisis ambientales, o bien provoca aquello que Eche- 
verría llama la "escasez artificial". Sin embargo, si bien Schmidt desarrolla una teoría sobre el concepto de naturaleza en Marx, no avanza hacia una teoría de la "forma natural" en tanto que modo de concreción de lo social dentro de el capitalismo.

Echeverría encuentra así un ámbito poco trabajado en el marxismo y que a la vez parece ser el elemento que hizo falta desarrollar en El capital de Marx. Por eso, la posibilidad de un marxismo contemporáneo radica en reconstruir una teoría de la forma natural, en tanto que discurso crítico negativo de la imperante forma de valor-trabajo del capitalismo: "si pensamos lo que sucede en realidad, el concepto de valor de uso que Marx opone al pensamiento moderno hace estallar el horizonte de inteligibilidad en el que éste se mueve" (Echeverría, 2001: 153).

\section{El valor de uso y la teoría de la forma natural}

Para Echeverría, ${ }^{5}$ poner en cuestión la figura que toma la reproducción social en el capitalismo a partir de la forma natural, significa cuestionar la relación de equilibrio entre el "sistema de necesidades" y "el sistema de capacidades". Para Marx, ésta era una forma de organización concreta de lo social en cuanto tiene como referente los bienes de consumo y los medios de producción. Por su parte, para Echeverría, esta definición expresaba que la forma natural es siempre un acto de transformación de lo "natural" hacia una forma "humana", de "trans-naturalización". Sin embargo, en el capitalismo, esta forma de organización de lo social a través de la producción y el consumo de bienes para la satisfacción de las necesidades y deseos de lo humano está subsumida siempre a una forma de

${ }^{5}$ La teoría de la forma natural, que en este artículo nos hemos propuesto extraer de su primer libro: El discurso crítico de Marx, sin duda es desarrollada con mayor amplitud en Valor de uso y utopía, así como en "Apuntes sobre la forma natural", en Modernidad y Blanquitud. 
organización social "abstracta", es decir, guiada por una lógica del tiempo de la valorización del valor. Esto devela que el capitalismo es una forma de reproducción social irracional. En primer lugar es tautológica, en segundo lugar responde a un telos ajeno a lo humano (el valor), y, en tercer lugar porque, para cumplir con este telos, es necesario depender de un sistema técnico altamente complejo y costoso, un sistema de reproducción altamente productivista, que, paradójicamente, no alcanza a establecer "una armonía" "entre el conjunto de capacidades y el conjunto de necesidades del sujeto social" (Echeverría, 2001: 172). A pesar del gran desarrollo técnico industrial que el capitalismo ha creado a lo largo de su historia y que en consecuencia ha permitido la gran producción de todo un "arsenal de mercancías", este complejo técnico no logra satisfacer las necesidades de lo social. Al contrario, este desequilibrio entre el sistema de las capacidades y el sistema de las necesidades trae consigo una constante "escasez artificial", que además de artificial, es necesaria, en la medida en que es arbitrariamente creada a pesar de que la producción de la riqueza esté en constante crecimiento, pues es indispensable para el cumplimiento de la reproducción en términos de lógica de la valorización. Esta escasez artificial tiene múltiples formas de concreción: las grandes expulsiones de seres humanos del ámbito del trabajo concentrados en la clase desempleada, grandes migraciones de individuos de la periferia hacia el centro; el desperdicio de enormes cúmulos de mercancía que no pudieron llegar a la esfera del consumo - un ejemplo magistral de este hecho son las nuevas ciudades construidas en China completamente abandonadas, a pesar de que las personas vivan hacinadas en pequeños cuartos en Beijing-y demás ejemplos que pueden ser sacados de la historia concreta de la reproducción social del capital.

Para Echeverría como para Marx, dichas manifestaciones de la contradicción capitalista están enraizadas en el proceso por el cual lo político o lo social está alienado. La implicación fundamental de 
la enajenación del trabajo en el capitalismo, que Marx desarrolla en El capital, está centrada en la idea de que esta enajenación no está limitada a un mero intercambio de salario por trabajo, sino que supone un proceso estructural a partir del cual la forma de reproducción social está subsumida al sujeto automático del capital, esto es, a la finalidad misma del proceso de producción/circulación capitalista, el cual es la valorización del valor o la acumulación. Para Echeverría, este proceso, además de subsumir materialmente el trabajo al capital, subsume, en consecuencia, la politicidad al capital; así Echeverría sostiene:

En el capítulo VI del primero libro de El capital..., Marx, sin mencionar el término 'enajenación', introduce su concepto en calidad de categoría central de la crítica de la economía política. Habla de valor de la mercancía capitalista como valor 'autovalorizándose' y lo califica de sujeto automático'... que organiza el conjunto de los actos de intercambio en la esfera de la circulación mercantil de la riqueza social... La reproducción mediante el mercado implica, por el contrario, la suspensión de la autarquía política del sujeto, la subordinación de su capacidad de autodefinirse prácticamente (Echeverría, 2017: 290-291).

El carácter fetichista de las relaciones sociales en el capital correspondería a la manifestación objetiva del proceso de enajenación de lo social en el capital, el cual debe reestructurar, de manera fetichista, la forma de lo político como ocurre de manera semejante con las relaciones sociales de producción; por ejemplo, a través de la cosificación del trabajo en la forma salario. De acuerdo con nuestro autor, el estado-nación capitalista sería la forma fetichista 
correspondiente al ámbito de lo político. ${ }^{6} \mathrm{~A}$ partir de esta base teórica, tres parecen ser los corolarios teóricos que se desprenden de la teoría de la forma natural.

En primer lugar, el capitalismo, como forma de reproducción concreta subsumida al principio abstracto de valorización del valor, provoca una sistemática "escasez artificial", tal como lo hemos ejemplificado más arriba.

En segundo lugar, después de haber desarrollado el concepto de enajenación más allá de los límites de la economía, el valor de uso y el ámbito de la forma natural se presentan como la esfera de lo posible, de la utopía y de la confrontación ante la ausencia de sentido del capital, a partir de una desestructuración del principio ético-económico que se impone con el valor: "a partir de este estudio, Echeverría aporta elementos para ver cómo esta cíclica es deconstruida por un valor de uso que, dentro de la enajenación capitalista, muestra, al deconstruir, desmontar y remontar el significado y el sentido de la vida, la posibilidad de una modernidad no capitalista" (Oliva, 2013: 61). ${ }^{7}$ Dicha idea es formulada en la teoría del cuádruple ethos moderno, cuando desarrolla las características de la modernidad barroca, como base para pensar una modernidad no-capitalista. En este sentido, la teoría del ethos barroco es un esfuerzo por comprender de qué manera la modernidad capitalista despliega su forma de reproducción social-natural en el contexto de las sociedades latinoamericanas y se manifiesta como una forma de desestructurar el principio ético-económico del capital. Con ethos Echeverría se refiere a un "uso, costumbre o comportamiento automático" que se presenta como un "principio

${ }^{6}$ Aquí no nos extendemos sobre este concepto pues supera el objetivo del artículo, sin embargo Echeverría desarrolla esta idea en su artículo "La nación posnacional" (Ver Bolívar, 2006).

${ }^{7}$ Para Carlos Oliva, en el espacio del valor de uso aún puede encontrarse cierto horizonte de sentido dentro de la barbarie capitalista. 
de construcción del mundo de la vida” (Echeverría, 1998: 37), por lo cual, podemos suponer que, a grandes rasgos, se refiere a la forma social-natural una vez que ha sido subsumida por la forma valor del capital. ${ }^{8}$ Nuestro autor propone cuatro $e$ the que se pueden reconocer en la historia de la modernidad capitalista: realista, romántico, clásico y barroco. El ethos realista para Echeverría se caracteriza por vivir el capitalismo desde una posición militante, afirmativa, esto es, asume el capitalismo como la mejor forma en la que se puede estructurar una sociedad gracias a la "pretensión de creatividad que tiene la acumulación del capital [...] y la pretensión de ésta no sólo de representar fielmente los intereses del proceso 'social-natural' de reproducción, sino de estar al servicio de la potenciación cuantitativa y cualitativa" (Echeverría, 1998: 38). Otro es el ethos romántico, igualmente militante, pero en el que se invierte la forma natural por la forma valor, negando así la contradicción intrínseca del capital: "confunde el 'valor de uso de las cosas' y las obras con su 'valor de cambio', pero en un peculiar sentido: afirma de manera absoluta el valor de uso" (Oliva, 2015: 146). En este ethos, después de negar la contradicción valor-valor de uso, se naturaliza la lógica misma de la relaciones sociales del capital. Por otra parte, el ethos clásico, a diferencia de los dos anteriores, no es militante, pero el enfrentamiento al capital está más allá del "margen de acción humano", en este, se afirma la inevitabilidad del capital como una necesidad trascendente y trágica; se aceptan las dificultades y las contradicciones que surgen del capitalismo, pero su solución estaría siempre enmarcada en el campo político de acción que el capitalismo ofrece, es decir, en el estado-nación capitalista a partir de una política reformista (Gandler, 2007: 415).

${ }^{8}$ Stefan Gandler sostiene al respecto: "Echeverría llega al análisis de las diversas modernidades capitalistas actuales que, a pesar de la 'pretensión de representación exclusiva de 'lo moderno' por parte de una de sus variantes, coexisten con ella" (371). 
El ethos barroco es una "estrategia de afirmación de la forma natural” (Echeverría, 1995: 165), pero en la que, a diferencia del ethos romántico, no se niega la existencia misma de la forma valor, sino que se reconoce el hecho de que el valor de uso está subsumido al valor de cambio. De manera que reinventa las cualidades de la forma social natural sobre la contradicción misma del capital, por ello es que en este ethos se encuentra la posibilidad de resistir a la enajenación estructural del capital, a través de la imposición de una lógica que trasciende a la valorización del valor, pero de segundo grado: "Esta barroca manera de ser moderno permite vivir la destrucción de lo cualitativo al convertirla en el acceso a la creación de otra dimensión, retadoramente imaginaria, de lo cualitativo" (Echeverría, 1998: 21).

En tercer lugar, para concluir, dentro del discurso marxista, la teoría de la forma natural pretende redefinir la teoría de lo político, más allá de la política del capitalismo. Como sostuvimos al principio, la teoría crítica tiene como tarea primordial ubicar el ámbito de la politicidad en la estructura capitalista de reproducción social. Sin embargo, el marxismo tradicionalmente ha dado por sentada la propuesta que Marx (2004) describe con respecto a lo político en su "Prólogo" a la Contribución a la crítica de la economía política:

En la producción social de su existencia, los hombres establecen determinadas relaciones, necesarias e independientes de su voluntad, relaciones de producción que corresponde a un determinado estadio evolutivo de sus fuerzas productivas materiales. La totalidad de esas relaciones de producción constituye la estructura económica de la sociedad, la base real sobre la cual se alza el edificio jurídico y político, y a la cual corresponden determinadas formas de consciencia social. El modo de producción material determina [bedingen] el proceso social, político e intelectual de la vida en general. No es la conciencia de los hombres lo que determina su 
ser, sino por el contrario, es su existencia social lo que determina su conciencia (66; las cursivas con mías).

Esta metáfora arquitectónica con respecto a la relación entre el espacio económico y espacio político ha sido tema de una larga discusión. La supuesta determinación de las relaciones económicas de producción sobre la "superestructura ideológica", parece conducir a lo siguiente: si la base económica es transformada, se transformará a su vez la superestructura ideológica. Al respecto, Karatani sostiene lo siguiente: "Esta visión pasa por alto el hecho de que el estado y la nación están enraizadas en la base estructural y por lo tanto son agentes activos" (2014: 3; la traducción es mía). En efecto, para Karatani, el capitalismo no sólo está concentrado en las relaciones de producción materiales, sino que conforma una alianza con la nación y con el estado, los cuales funcionan como elementos activos del sistema de reproducción social y de la afirmación del poder, el cual se define más que como un modo de producción material, como un modo de intercambio donde cada una de estas instituciones entablan, en un sentido amplio de la economía, un tipo de intercambio social.

Desde la perspectiva de Echeverría, la definición de lo político en los términos de la superestructura resulta insuficiente para el discurso crítico: "La infraestructura es aquí la forma burguesa de la sociedad económica o no reflexiva; igualmente, la superestructura es aquí la forma burguesa de la sociedad política o reflexiva. Estas formulaciones del 'Prólogo' presentan una insuficiencia si se quiere desarrollar exhaustivamente a partir de ellas una crítica radical de la política capitalista” (2017: 295). El proyecto echeverriano, el cual hemos tratado de desarrollar en este artículo, se define en consecuencia en los siguientes términos: "Podría decirse que esta insuficiencia tradicional del 'Prólogo' de 1859 proviene de la utilización de la oposición tradicional entre economía y política allí 
donde el nuevo concepto crítico de infraestructura como el lugar del 'conflicto entre fuerzas productivas sociales y condiciones de producción' requeriría ser desarrollado en los términos de $E l$ capital' (Echeverría, 2017: 295). En efecto, habrá, en cambio, que volver a la base de la problemática misma de este libro: la contradicción entre el valor y el valor de uso, que hemos señalado más arriba. Así, concluye Echeverría al respecto: "Y uno de los resultados principales es, sin duda, el descubrimiento de que lo político, aún en su forma capitalista enajenada, es el carácter fundamental de la infraestructura, del proceso de reproducción de la riqueza social" (2017: 295).

El estado en su forma burguesa-capitalista no abarca todo el espacio de lo político, sino que se encarga simplemente en términos de la economía de administrar los "resultados" de la enajenación. En cambio, este espacio llamado "forma social-natural", esta contraparte concreta de la forma de reproducción social, es para Echeverría el espacio en el que propiamente se juega la política, a diferencia del espacio de la "sociedad civil", en el que se despliega lo político en su forma capitalista. En efecto, la forma natural significa ese momento social en el que al reproducirse la vida concreta de una sociedad histórica particular, se juega en ella la posibilidad "democrática", autárquica y autónoma de definirse a sí misma, es decir, de darse a sí misma una forma concreta y simbólica, de darse una identidad (Echeverría, 2010: 111). ${ }^{9}$

${ }^{9}$ Este planteamiento lo sostenía Echeverría desde El discurso crítico de Marx y es retomado por muchos de los marxistas que reflexionan sobre el desarrollo del capitalismo en su última fase, la neoliberal. Como sostuvimos al principio del artículo, los marxismos contemporáneos tales como el marxismo ecológico desarrollado por John Bellamy Foster y su teoría de la fractura metabólica retoma el lugar de la forma natural y del valor de uso como punto políticos de la lucha: "es a través de la politización de la estructura del valor de uso de la economía, y su relación con el proceso de trabajo y con toda la estructura cualitativa de la 


\section{Bibliografía}

Echeverría, Bolívar, 1995, Las ilusiones de la modernidad, Universidad Nacional Autónoma de México/El equilibrista, México.

, 1998, La modernidad de lo barroco, Era, México.

, 2001, Valor de uso y utopia, Siglo XXI, México.

, 2010, Modernidad y Blanquitud, Era, México.

, 2017, El discurso critico de Marx, Fondo de Cultura Económica/Itaca, México.

Foster, John Bellamy, 2004, La ecología de Marx, materialismo y naturaleza, Carlos Martín y Carmen González (trad.), Intervención cultural/El Viejo Topo, Madrid.

Foster, John Bellamy , 2013, "Marx and the Rift in the Universal Metabolism of Nature", Monthly Review Press, Nueva York. Versión en español disponible en: https://marxismocritico. com/2014/12/23/marx-y-la-fractura-en-el-metabolismo-universal-de-la-naturaleza/ (consultado: 18/11/2019)

Gandler, Stefan, 2007, Marxismo crítico en México: Adolfo Sánchez Vázquez y Bolivar Echeverría, Fondo de Cultura Económica, México.

Gorz, André, 2011, Ecológica, Pablo Betesh (trad.), Capital Intelectual, Buenos Aires.

Karatani, Kojin, 2004, Transcritique, Sabu Koshu (trad.), MIT Press, Cambridge.

, 2014, The structure of world history, Michael K. Bourdaghs (trad.), Duke Press, Durham.

economía, que el abordaje dialéctico de Marx en el metabolismo entre la naturaleza y la sociedad asume una forma potente" (Foster, 2014). 
Marcuse, Herbert, 1972, Razón y revolución, Alianza editorial, Madrid.

Marx, Karl,1973, Elementos fundamentales para la crítica de la economía política, LI, Siglo XXI, México.

, 1976, "Glosas marginales al 'tratado de economía política' de Adolph Wagner”, en Maurice Dobb, Estudios sobre el capital, José Aricó (trad.), Siglo XXI, Madrid.

, 2001, Capitulo VI (inédito), resultados del proceso inmediato de producción, Pedro Scaron (trad., y notas), Siglo XXI, México.

, 2001, El capital, critica de la economía politica, Tomo I, Siglo XXI, México.

, 2004, Introducción general a la critica de la economía politica /1857, Siglo XXI, México.

Oliva Mendoza, Carlos, 2013, Semiótica y capitalismo, Universidad Nacional Autónoma de México/Ítaca, México.

,2015, "La época romántica de la poesía mexicana”, en Rogelio Guedea (coord.), Historia critica de la poesía mexicana, t. I, Fondo de Cultura Económica, México.

Ortega Reyna, Jaime, 2014, "El valor de uso de Bolívar Echeverría”, en David Arredondo y Jaime Ortega Reyna (comps.), Pensamiento nuestroamericano, Universidad Nacional Autónoma de México/Estudios latinoamericanos, México, pp. 14-40.

Roldosky, Roman, 1968, Génesis y estructura de El capital, León Mames (trad.), Siglo XXI, México.

Schmidt, Alfred, 1975, Feuerbach o la sensibilidad emancipada, Taurus, México. xico.

,2014, El concepto de naturaleza en Marx, Siglo XXI, Mé- 
Bibliografía secundaria con respecto a la teoría del valor de uso/forma natural de Echeverría:

Fuentes Diana, Isaac Venegas y Carlos Oliva (comps.), 2012, Bolivar Echeverría: Crítica e interpretación, Ítaca/Universidad Nacional Autónoma de México, México,

Gandler, Stefan, 2016, Teoría critica, imposible resignarse, Pesadillas de represión y aventuras de emancipación, Miguel Ángel Porrúa editor/Universidad Autónoma de Querétaro, México.

Inclán, Daniel, Márgara Millán y Lucia Linsalata, 2012,"Apuesta por el valor de uso: aproximación a la arquitectónica del pensamiento de Bolívar Echeverría", Íconos, vol. 16, núm. 2, Facultad Latinoamericana de Ciencias Sociales, Ecuador, pp. 19-32.

Morańa, Mabel, 2014, Para una crítica de la modernidad capitalista, Dominación y resistencias en Bolivar Echeverría, DGE/Equilibrista, Quito.

Serur Smeke, Raquel (comp.), 2015, Bolivar Echeverría modernidad y resistencias, Universidad Autónoma Metropolitana/Era, México. 